\title{
A Low-cost Optimization Design for Minimizing Chromatic Aberration by Doublet Prisms
}

\author{
Wen-Shing Sun ${ }^{1}$, Chuen-Lin Tien ${ }^{2 *}$, Ching-Chemg Sun ${ }^{1}$, and Ching-Chun Lee ${ }^{1}$ \\ ${ }^{I}$ Department of Optics and Photonics, National Central University, Chung-Li 32001, Taiwan, R.O.C. \\ ${ }^{2}$ Department of Electrical Engineering, Feng Chia University, Taichung 40724, Taiwan R.O.C.
}

(Received June 20, 2012 : revised September 5, 2012 : accepted September 26, 2012)

\begin{abstract}
A low-cost optimal double-prism method is proposed by using the developed MATLAB program to correct chromatic aberration. We present an efficient approach to choose a couple of low-cost glasses to obtain a low aberration double prism. The doublet prisms were made of two lead-free glasses. The relative partial dispersion of the two lead-free glasses is identical and their Abbe numbers are different greatly. The proposed design aims to minimize chromatic aberration, such as in apochromats, for paraxial ray tracing. Finally, an optimization design for real ray tracing can be evaluated by the chromatic aberration curve with a minimal area.
\end{abstract}

Keywords : Doublet prisms, Optimization, Chromatic aberration, Ray tracing

OCIS codes : (080.1010) Aberrations; (080.2740) Geometric optical design; (230.5480) Prisms

\section{INTRODUCTION}

The image quality of a lens design can be good but the cost of the glass from which it is made can be quite high. Kidger [1] considered variation in cost between glasses used for this purpose. In the large size lenses, the cost of expensive glass could be prohibitive; but in small size lenses, the materials make up only a small percentage of the total cost of the lens, so expensive materials might well be acceptable in this case. Chromatic aberration occurs because of the different refractive indices of lenses for different wavelengths of light $[2,3]$. If two different types of glasses are combined into a thin two-element system, a paraxial chromatic aberration will develop [4]. Robb [5] developed a method using two different types of glass for the correction of axial color for at least three wavelengths. Certain combinations might be found for correction at four and five wavelengths. In 1983, Sharma and Gopal [6] used the double-graph technique to produce doublet designs. Then, in 2001, Rayces and Rosete-Aguilar [7, 8] described a method to select pairs of glasses for both thin cemented achromatic doublets and thin aplanatic achromatic doublets with a reduced secondary spectrum. In another study, Banerjee and Hazra [9] used a genetic algorithm for the structural design of cemented doublets. The aim of this work is to minimize chromatic aberration by using a low-cost optimal double-prism method. An efficient approach for finding an optimum design is also proposed.

\section{METHODS}

An illustration of ray tracing in and out of a prism is shown in Fig. 1. The angle of a ray in the normal direction from the prism surface is positive in the anticlockwise

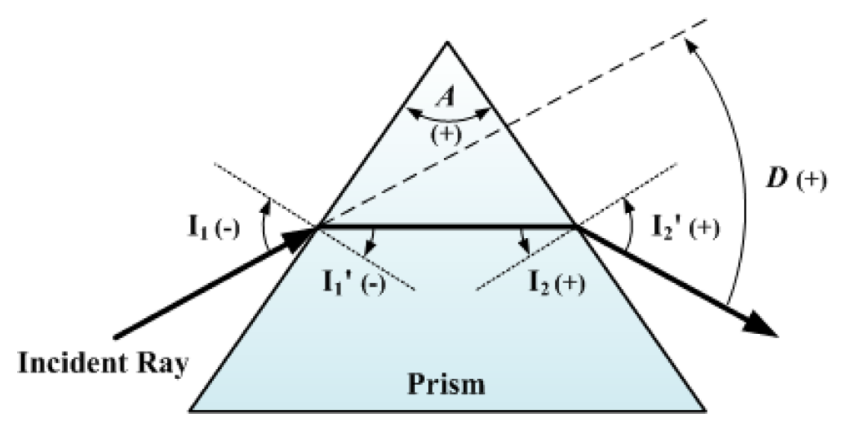

FIG. 1. Angle of deviation of a prism.

\footnotetext{
*Corresponding author: cltien@fcu.edu.tw

Color versions of one or more of the figures in this paper are available online.
} 
direction, and negative in the clockwise direction. In this figure, $I_{1}$ and $I_{2}$ are the incident angles of the first and the second surfaces, respectively; $I_{1}^{\prime}$ and $I_{2}^{\prime}$ are the refractive angles of the first and the second surfaces, respectively; $A$ is the apex angle of the prism. The sign of the apex angle is positive in the vertical, and negative in the inverse direction. The ray thus deviates through an angle of $\left(I_{l}{ }^{\prime}-I_{l}\right)$ at the first surface. At the second surface, the ray deviates by $\left(I_{2}^{\prime}-I_{2}\right)$, so the angle of deviation $D$ of the ray is given by

$$
D=\left(I_{1}^{\prime}-I_{1}\right)+\left(I_{2}^{\prime}-I_{2}\right)
$$

If we consider real ray tracing, the deviation angle [10] presented by

$$
D=-I_{1}-A+\sin ^{-1}\left(\left(n^{2}-\sin ^{2} I_{1}\right)^{1 / 2} \sin A+\cos A \sin I_{1}\right)
$$

\subsection{Single Prism Paraxial Chromatic Aberration}

If all the angles of the design are small, the equations for the paraxial ray tracing can be obtained, and the paraxial deviation angle [11] given by

$$
D=(n-1) A .
$$

The paraxial primary color $\mathcal{E}_{F, C}$ is the difference in deviation angles between $\mathrm{F}$ line $(0.48613 \mu \mathrm{m})$ and $\mathrm{C}$ line $(0.65627 \mu \mathrm{m})$ as shown. Thus

$$
\varepsilon_{F, C}=D_{F}-D_{C}=\frac{D_{d}}{V_{d}},
$$

where $D_{d}$ is the paraxial deviation angle of d line $(0.58756$ $\mu \mathrm{m}) ; V_{d}=\left(n_{d}-1\right) /\left(n_{F}-n_{C}\right)$ is the Abbe number; and $n_{C}, n_{d}$, $n_{F}$ are the refractive indices of $\mathrm{C}, \mathrm{d}$, and $\mathrm{F}$ lines, respectively. Consequently, the paraxial second spectrum $\mathcal{E}_{d, C}$ is the difference in the deviation angles between $\mathrm{d}$ line and $\mathrm{C}$ line. This can show as

$$
\varepsilon_{d, C}=D_{d}-D_{C}=\frac{P_{d, C}}{V_{d}} D_{d},
$$

where $P_{d, C}=\left(n_{d}-n_{C}\right) /\left(n_{F}-n_{C}\right)$ is the relative partial dispersion.

\subsection{Doublet Prisms Paraxial Chromatic Aberration}

In the paraxial ray tracing of doublet prisms, the angle of deviation of d-line light is defined as $3^{\circ}$, so the primary color $\varepsilon_{F, C}$ is zero $[4,5]$. These equations can be written as

$$
\begin{aligned}
& \mathrm{D}_{d}=D_{d_{1}}+D_{d_{2}}=3^{\circ}, \\
& \varepsilon_{F, C}=\frac{D_{d_{1}}}{V_{d_{1}}}+\frac{D_{d_{2}}}{V_{d_{2}}}=0,
\end{aligned}
$$

where the paraxial deviation angles of the doublet prisms are $D_{d 1}$ and $D_{d 2}$, respectively. The primary chromatic aberration is $\mathcal{E}_{F, C}$. Solving for $D_{d l}$ and $D_{d 2}$ from the above equations, we obtain

$$
D_{d_{1}}=\frac{V_{d_{1}}}{V_{d_{1}}-V_{d_{2}}} D_{d}
$$

and

$$
D_{d_{2}}=\frac{-V_{d_{2}}}{V_{d_{1}}-V_{d_{2}}} D_{d}
$$

The apex angles of the doublet prisms are expressed as

$$
A_{1}=\frac{D_{d_{1}}}{n_{d_{1}}-1},
$$

and

$$
A_{2}=\frac{D_{d_{2}}}{n_{d_{2}}-1} .
$$

Accordingly the paraxial primary color is zero, but there are still some paraxial secondary spectra. Thus the paraxial secondary spectrum can be defined as

$$
\begin{aligned}
\varepsilon_{d, C} & =\varepsilon_{d, C_{1}}+\varepsilon_{d, C_{2}} \\
& =\frac{P_{d, C_{1}}}{V_{d_{1}}} D_{d_{1}}+\frac{P_{d, C_{2}}}{V_{d_{2}}} D_{d_{2}} . \\
& =\frac{\left(P_{d, C_{1}}-P_{d, C_{2}}\right)}{\left(V_{d_{1}}-V_{d_{2}}\right)} D_{d}
\end{aligned}
$$

where the paraxial primary chromatic aberrations of the doublet prisms are $\mathcal{E}_{F, C l}$ and $\mathcal{E}_{F, C 2}$, respectively.

\subsection{Schott Glasses Selection}

We choose the Schott glass [12] for the design because of the large number of types of glasses that are available. The Abbe numbers of the different glasses have been ranked. There are 119 different optical glasses all with different prices. The price of N-BK7 is the lowest. The relative price (RP) is found by comparison and the results are indexed. To avoid using the most expensive types of glass in the design, the twenty-nine types with a relative price $\mathrm{RP} \geq 17$ as well as those with no marked prices are eliminated. The costs of glasses such as N-KZFS11, N-PK51, and N-LASF31A, are much higher than the others. Those with no marked prices are molding glasses or new types of glasses. The internal transmittance is the transmittance of light excluding reflection loss. The N-SF6HT and N-SF57HT glasses offer improved transmittance in the 
visible spectral range especially in the blue-violet area. Moreover, since the $V_{d}, n_{d}$, and $P_{d, C}$ of N-SF6HT and $\mathrm{N}$-SF57HT are all the same as those of N-SF6 and $\mathrm{N}-\mathrm{SF} 57$, the corrected chromatic aberrations will be almost the same. Thus we can neglect the N-SF6HT and $\mathrm{N}-\mathrm{SF} 57 \mathrm{HT}$ glasses. A total of seventy types of glasses were chosen for doublet prisms design to correct chromatic aberration.

\subsection{Merit Function}

In Eqs. (2) and (3), it can be seen that the there is a difference in the deviation angles between the real ray and the paraxial ray. The deviation angle of the paraxial ray is unconcerned with the incident angle $I_{1}$, but the deviation angle of the real ray is related to the incident angle $I_{1}$. When the incident angle increases, the real and paraxial chromatic aberrations will be very different. The real chromatic aberration is corrected for optimization. The damped least-squares method [13-15] is applied for an optimization design of the chromatic aberration. A merit function is defined as the summation of the squared values of the weighting differences between the aberrations and their target values. The formula can be written as

$$
\phi=\sum_{i=1}^{m} w_{i}^{2}\left(e_{i}-t_{i}\right)^{2}
$$

where $m$ is the total summation number; the $w_{i}$ is the weighting factor; $e_{i}$ is the aberration and $t_{i}$ is the target value. We define the function $f_{i}\left(x_{1}, x_{2}, \cdots, x_{n}\right)$ as

$$
f_{i}=w_{i}\left(e_{i}-t_{i}\right) .
$$

Before optimization, the $n$ variables are denoted as $x_{10}$, $x_{20}, \cdots, x_{n} 0$; the $m$ aberrations before the optimization are $f_{10}, f_{20}, \cdots, f_{m 0}$. After the optimization process, the variables are denoted as $x_{1}, x_{2}, \cdots, x_{n}$, and the aberrations as $f_{1}, f_{2}$, $\cdots ., f_{m}$. Here, we define a matrix $A$, in which the elements are

$$
A i j=\frac{\partial f_{i}}{\partial x_{j}}
$$

We then get the equation

$$
\mathrm{X}=-\left(A^{T} A+p I\right)^{-1} A^{T} f_{0},
$$

where $A^{T}$ is the transpose matrix of $A$; $I$ is a unit matrix, $p$ is a damping factor; and $f_{0}$ is the matrix containing the elements $f_{10}, f_{20}, \cdots . f_{m 0}$. If $x$ and $x_{0}$ are the matrices containing the elements $x_{1}, x_{2}, \cdots, x_{n}$, and $x_{10}, x_{20}, \cdots, x_{n}$, respectively, we can obtain

$$
x=x_{0}+\mathrm{X} .
$$

\section{RESULTS}

\subsection{Minimizing the Paraxial Chromatic Aberration}

The doublet prisms have two apex angles. The angle of deviation of $\mathrm{d}$ line is $3^{\circ}$, and the primary color is eliminated. The steps are repeated to reduce the secondary spectra of the doublet prisms. Using Eq. (12), the correct doublet prisms combination can be found by choosing the smaller $\left(P_{d C_{1}}-P_{d C_{2}}\right)$ and the larger $\left(V_{d_{1}}-V_{d_{2}}\right)$. When the $\left(P_{d C_{1}}-P_{d C_{2}}\right) /\left(V_{d_{1}}-V_{d_{2}}\right)$ is close to zero, the chromatic aberration is smaller. Figure 2 shows the relative partial dispersion with respect to the $V_{d}$ number. We chose six groups from $\mathrm{A}$ to $\mathrm{F}$ for minimizing the paraxial chromatic aberration. The design results are listed in Table 1, where the $\mathrm{CA}$ is the area of the chromatic aberration curve. Figure 3 shows the chromatic aberration curves. The

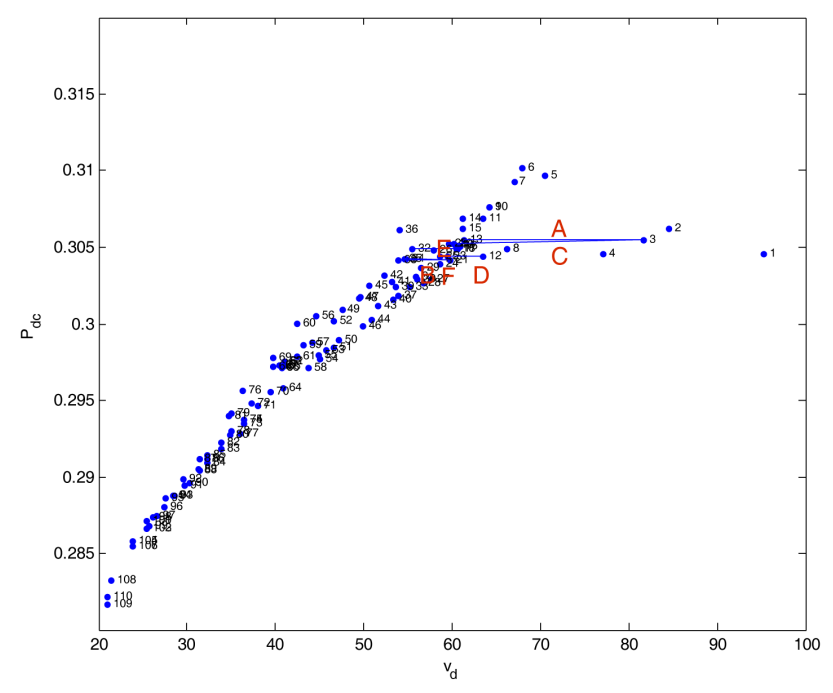

FIG. 2. $P_{d, C}-V_{d}$ mapping for the selected doublet prisms.

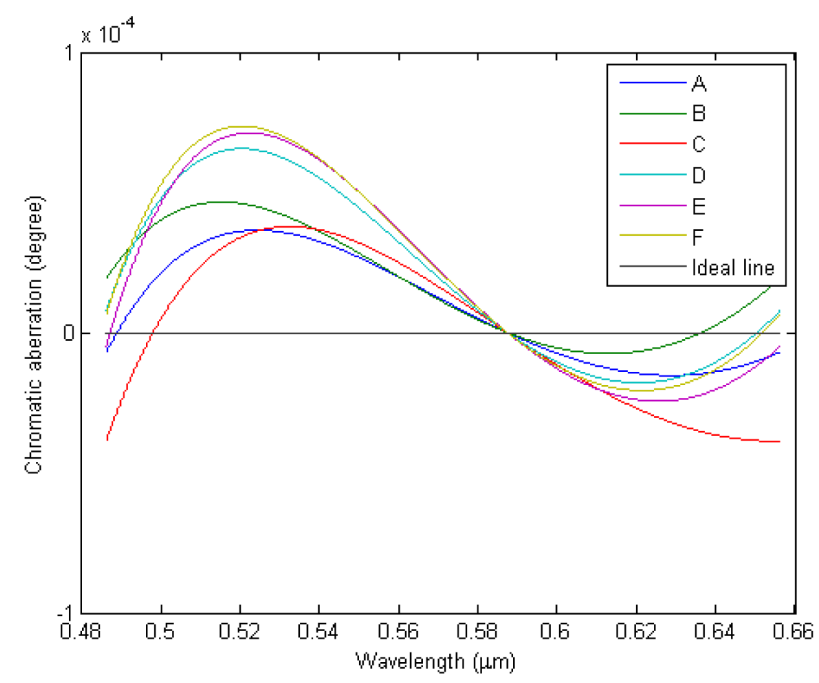

FIG. 3. Minimizing paraxial chromatic aberration curves for doublet prisms groups $\mathrm{A}$ to $\mathrm{F}$. 
TABLE 1. Design data and area of paraxial chromatic aberration curves for doublet prisms A To F

\begin{tabular}{c|c|c|c|c|c|c}
\hline \hline \multicolumn{2}{c|}{$D_{\mathrm{d}}=3^{\circ}, E_{\mathrm{f}, \mathrm{C}}=0$} \\
No. & Glass 1 (No.) & Glass 2 (No.) & $\begin{array}{c}A_{1} \\
\text { Degree }\end{array}$ & $\begin{array}{c}A_{2} \\
\text { Degree }\end{array}$ & $\begin{array}{c}E_{d, C} \\
\text { Degree }\end{array}$ & $\begin{array}{c}\text { CA } \\
\mu \mathrm{m} \times \text { Degree }\end{array}$ \\
\hline A & N-Pk52A(3) & N-Sk5(13) & 24.215 & -15.336 & $7.092 \times 10^{-6}$ & $3.029 \times 10^{-6}$ \\
\hline B & N-Bak2(21) & N-Lak34(35) & 63.681 & -43.043 & $-1.916 \times 10^{-5}$ & $3.432 \times 10^{-6}$ \\
\hline C & N-Pk52A(3) & N-Lak21(20) & 22.903 & -13.088 & $3.870 \times 10^{-5}$ & $4.165 \times 10^{-6}$ \\
\hline D & N-Psk53A(12) & N-Lak7(25) & 63.161 & -55.300 & $-7.641 \times 10^{-6}$ & $4.952 \times 10^{-6}$ \\
\hline E & N-Sk14(17) & N-Lak14(32) & 58.115 & -45.996 & $5.195 \times 10^{-6}$ & $5.612 \times 10^{-6}$ \\
\hline F & N-Bak2(21) & N-Lak8(38) & 56.447 & -38.540 & $-6.270 \times 10^{-6}$ & $5.568 \times 10^{-6}$ \\
\hline
\end{tabular}

TABLE 2. Initial values for optimization of the doublet prism design (group A)

\begin{tabular}{c|c|c|c|c|c|c|c}
\hline \hline \multicolumn{10}{c}{ N-PK52A (3), N-SK5 (13) } \\
\hline $\begin{array}{c}\mathrm{I}_{1} \\
\text { degree }\end{array}$ & $\begin{array}{c}\mathrm{I}_{3} \\
\text { degree }\end{array}$ & $\begin{array}{c}A_{1} \\
\text { degree }\end{array}$ & $\begin{array}{c}A_{2} \\
\text { degree }\end{array}$ & $\begin{array}{c}D_{d} \\
\text { degree }\end{array}$ & $\begin{array}{c}\mathcal{E}_{d, C} \\
\text { degree }\end{array}$ & $\begin{array}{c}\mathcal{E}_{F, C} \\
\text { degree }\end{array}$ & $\begin{array}{c}\mathrm{CA} \\
\mu \mathrm{m} \times \text { degree }\end{array}$ \\
\hline 0 & 0 & 24.215 & -15.336 & 4.148 & $6.332 \times 10^{-3}$ & $2.078 \times 10^{-2}$ & $9.083 \times 10^{-4}$ \\
\hline
\end{tabular}

horizontal ideal line, which denotes the angle of deviation of $\mathrm{d}$ line is $3^{\circ}$, has been set to zero for the chromatic aberration. The other lines are described as the chromatic aberration of doublet prisms groups from $\mathrm{A}$ to $\mathrm{F}$.

\subsection{OPtimization Design For The Real Chromatic Aberration}

We choose group A from Table 1 as an example as the initial value. The doublet prisms are made of N-PSK52A and N-SK5 glasses. We set $I_{1}$ and $I_{3}$ as the incident angles for the first surface of the first and the second prisms, respectively; $A_{1}$ and $A_{2}$ are the apex angles of the first and the second prisms, respectively. An illustration of the ray tracing of the doublet prisms is shown in Fig. 4. At the initial values, we set $I_{1}=0, I_{3}=0, A_{1}=24.215^{\circ}$, and $A_{2}=-15.336^{\circ}$, as listed in Table 2 . We can calculate the $D_{d}=4.148^{\circ}, \varepsilon_{d, C}=6.332 \times 10^{-3}, \varepsilon_{F, C}=2.078 \times 10^{-2}$, and $\mathrm{CA}=$ $9.083 \times 10^{-4}$ for the real ray tracing. The $D_{d}$ and chromatic aberrations between the real and the paraxial rays $\left(\varepsilon_{F, C}\right.$, $\left.\varepsilon_{d, C}, \mathrm{CA}\right)$ are very different. The real chromatic aberration is corrected by an optimization program.

The merit function consists of three terms. The first term is the deviation angle $D_{d}$ of the real ray for the doublet prisms, the second is the real primary color aberration $\varepsilon_{F, C}$ of the doublet prisms, and the last is the real secondary spectrum $\varepsilon_{d, C}$ of the doublet prisms. If the target values are $t_{D_{d}}=3, t_{\varepsilon_{d, C}}=0$, and $t_{\varepsilon_{F, C}}=0$, then the merit function is given by

$$
\phi=\sum_{i=1}^{3} f_{i}^{2}=w_{1}^{2}\left(D_{d}-3\right)^{2}+w_{2}^{2}\left(\varepsilon_{d, C}-0\right)^{2}+w_{3}^{2}\left(\varepsilon_{F, C}-0\right)^{2},
$$

where the weighting factors are $w_{1}=1, w_{2}=20, w_{3}=20$, respectively. During the doublet prisms optimization, we

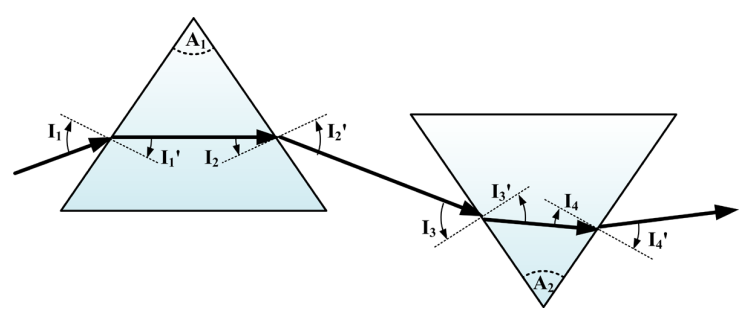

FIG. 4. Ray tracing in the doublet prisms.

will consider some sort of aberration balance, a sensible choice of weighting factors is essential if we are to achieve the best possible performance. In the optimization process, we think that two aberrations of $\varepsilon_{d, C}$ and $\varepsilon_{F, C}$ are more rigorous than that of $D_{d}$, and then the target values of both $\left|\varepsilon_{d, C}\right|$ and $\left|\varepsilon_{F, C}\right|$ are twenty times smaller than those of $\left|D_{d}-3\right|$. It is therefore sensible, often but not always, for weighting factors to be smaller for larger target values.

We use four variables as $x_{1}, x_{2}, x_{3}$, and $x_{4}$, to represent the incident angle $I_{1}$ of the first prism, the incident angle $I_{3}$ of the second prism, the apex angle $A_{1}$ of the first prism, the apex angle $A_{2}$ of the second prism, respectively. Before optimization, the variables are denoted as $x_{10}, x_{20}$, $x_{30}$, and $x_{40}$, which correspond to $I_{1}=0, I_{3}=0, A_{1}=24.215$, and $A_{2}=-15.336$, respectively. The optimization results are listed in Table 3 , and the real chromatic aberration curve is shown in Fig. 5.

Except for fixing the deviation angle of real ray $D_{d}=$ $3^{\circ}$, we can optimize the area $\mathrm{CA}$ of the real chromatic aberration curve to obtain an optimization design. The merit function is defined as

$$
\phi=w_{1}^{2}\left(D_{d}-t_{D_{d}}\right)^{2}+w_{2}^{2}\left(C A-t_{C A}\right)^{2},
$$


TABLE 3. Design results (group A) for target values: $t_{D_{d}}=3, t_{\epsilon_{d C}}=0$, and $t_{\epsilon_{F C}}=0$

\begin{tabular}{c|c|c|c|c|c|c|c}
\hline \hline \multicolumn{10}{c}{ N-PK52A(3), N-SK5(13) } \\
\hline $\begin{array}{c}\mathrm{I}_{1} \\
\text { degree }\end{array}$ & $\begin{array}{c}\mathrm{I}_{3} \\
\text { degree }\end{array}$ & $\begin{array}{c}A_{1} \\
\text { degree }\end{array}$ & $\begin{array}{c}A_{2} \\
\text { degree }\end{array}$ & $\begin{array}{c}D_{d} \\
\text { degree }\end{array}$ & $\begin{array}{c}\varepsilon_{d, C} \\
\text { degree }\end{array}$ & $\begin{array}{c}\varepsilon_{F, C} \\
\text { degree }\end{array}$ & $\begin{array}{c}\text { CA } \\
\mu \mathrm{m} \times \text { degree }\end{array}$ \\
\hline-6.4399 & -0.30885 & 23.9821 & -15.6298 & 3 & $2.873 \times 10^{-6}$ & $-8.664 \times 10^{-7}$ & $3.214 \times 10^{-6}$ \\
\hline
\end{tabular}

TABLE 4. Design results (group A) for target values: $t_{D_{d}}=3, t_{C A}=0$

\begin{tabular}{c|c|c|c|c|c|c|c}
\hline \hline \multicolumn{1}{c}{ N-PK52A(3), N-SK5(13) } \\
\hline $\begin{array}{c}\mathrm{I}_{1} \\
\text { degree }\end{array}$ & $\begin{array}{c}\mathrm{I}_{3} \\
\text { degree }\end{array}$ & $\begin{array}{c}A_{1} \\
\text { degree }\end{array}$ & $\begin{array}{c}A_{2} \\
\text { degree }\end{array}$ & $\begin{array}{c}D_{d} \\
\text { degree }\end{array}$ & $\begin{array}{c}\varepsilon_{d, C} \\
\text { degree }\end{array}$ & $\begin{array}{c}\varepsilon_{F, C} \\
\text { degree }\end{array}$ & $\begin{array}{c}\text { CA } \\
\mu \mathrm{m} \times \text { degree }\end{array}$ \\
\hline-6.6347 & -0.29965 & 23.9982 & -15.6213 & 3 & $-1.430 \times 10^{-5}$ & $-5.786 \times 10^{-5}$ & $1.702 \times 10^{-6}$ \\
\hline
\end{tabular}

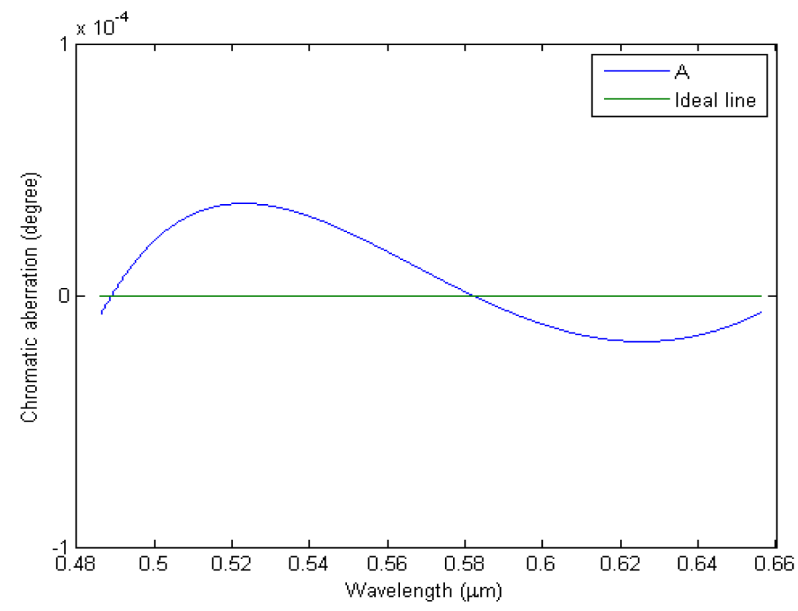

FIG. 5. Real chromatic aberration curve for group A for optimized target values: $t_{D_{d}}=3, t_{\epsilon_{d, C}}=0$, and $t_{\epsilon_{F, C}}=0$.

where the target values are $t_{D_{d}}=3$, and $t_{C A}=0$. The optimized results are listed in Table 4 , and the real chromatic aberration curve is shown in Fig. 6.

\subsection{Total Intemal Reflection}

As mentioned before, figure 4 shows the ray tracing of the doublet prisms, where $I_{1}, I_{1}{ }^{\prime}, A_{2}, I_{4}$, and $I_{4}{ }^{\prime}$ are negative and $A_{1}, I_{2}, I_{2}{ }^{\prime}, I_{3}$, and $I_{3}^{\prime}$ are positive. When the incident angle of the first prism is $I_{2}>\theta_{C}$, the total internal reflection of the ray appears. The critical angle $\theta_{C}$ is given by

$$
\theta_{C}= \pm \sin ^{-1} \frac{1}{n}
$$

where $n$ is the refractive index of the prism.

We choose group B from Table 5 as an example. The two types of glass used in the doublet prisms are N-BAK2 and N-LAK34, their refractive indices are $n_{d_{1}}=1.53996$ and $n_{d_{2}}=1.72916$, respectively, and the critical angles are $\theta_{C_{1}}$ $=40.494^{\circ}$ and $\theta_{C_{2}}=-35.332^{\circ}$, respectively. In order to avoid total reflection, the incident angle $I_{1}$ of the first surface of

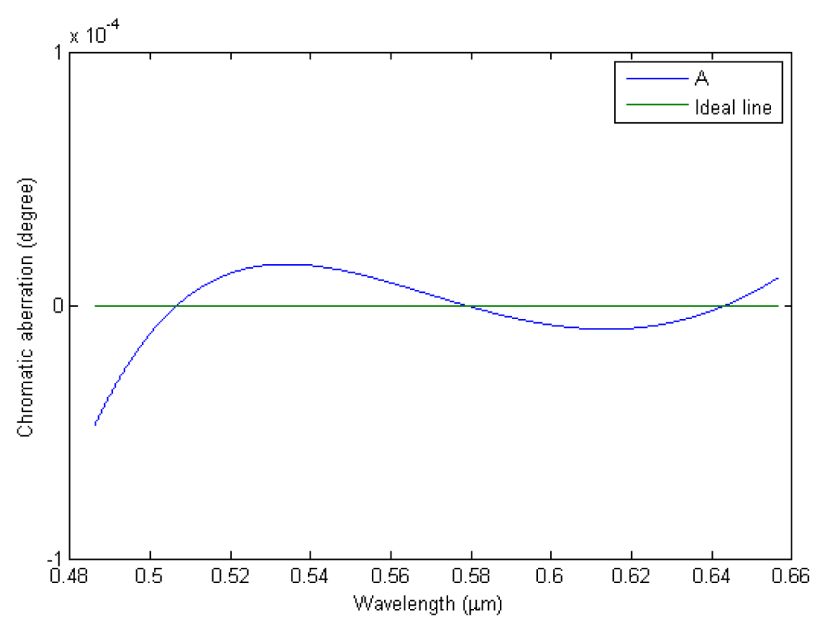

FIG. 6. Real chromatic aberration curve for group A for optimized target values: $t_{D_{d}}=3, t_{C A}=0$.

the first prism must be

$$
I_{1}<\sin ^{-1}\left[n_{1} \sin \left(\theta_{C_{1}}-A_{1}\right)\right]
$$

and the incident angle $I_{3}$ of the first surface of the second prism is required to be

$$
I_{3}<\sin ^{-1}\left[n_{2} \sin \left(\theta_{C_{2}}-A_{2}\right)\right] .
$$

\subsection{Design of The Optimization Program}

A flow-chart of the optimization program for the doublet prisms design is shown in Fig. 7. First, the program selects $\left(P_{d C_{1}}-P_{d C_{2}}\right) /\left(V_{d_{1}}-V_{d_{2}}\right)$, the minimal value of the doublet glasses. Second, the program uses the paraxial ray equations from Eq. (6) to Eq. (11) to fix the deviation angle $D_{d}=3^{\circ}$, and eliminate the primary color. Then, the two apex angles are obtained. Third, the program sets $t_{D_{d}}=3, t_{\varepsilon_{d C}}=0$, and $t_{\varepsilon_{F C}}=0$ to optimize the real primary color. Finally, $t_{D_{d}}=3$ and $t_{C A}=0$ are used to optimize the area of the real chromatic aberration curve, until the real chromatic aberration is a minimum. The 
TABLE 5. Optimization designs for doublet prisms from group A to group F

\begin{tabular}{c|c|c|c|c|c|c|c}
\hline \multicolumn{7}{c}{$D_{\mathrm{d}}=3^{\circ}$} \\
\hline NO. & Glass 1 & Glass 2 & $\begin{array}{c}I_{I} \\
\text { degree }\end{array}$ & $\begin{array}{c}I_{3} \\
\text { degree }\end{array}$ & $\begin{array}{c}A_{1} \\
\text { degree }\end{array}$ & $\begin{array}{c}A_{2} \\
\text { degree }\end{array}$ & $\begin{array}{c}\text { CA } \\
\mu \mathrm{m} \times \text { degree }\end{array}$ \\
\hline A & N-PK52A & N-SK5 & -6.6347 & -0.29965 & 23.9982 & -15.6213 & $1.702 \times 10^{-6}$ \\
\hline B & N-BAK2 & N-LAK34 & -50.1947 & 1.1397 & 15.8195 & -12.3902 & $4.405 \times 10^{-6}$ \\
\hline C & N-PK52A & N-LAK21 & 3.0797 & 0.17857 & 22.7408 & -14.9452 & $2.699 \times 10^{-6}$ \\
\hline D & N-PSK53A & N-LAK7 & -48.8909 & -0.54463 & 14.5904 & -14.1276 & $4.468 \times 10^{-6}$ \\
\hline E & N-SK14 & N-LAK14 & -47.9023 & 0.16197 & 15.4029 & -13.4042 & $4.949 \times 10^{-6}$ \\
\hline F & N-BAK2 & N-LAK8 & -49.0944 & 1.0355 & 16.0228 & -12.4993 & $4.729 \times 10^{-6}$ \\
\hline
\end{tabular}

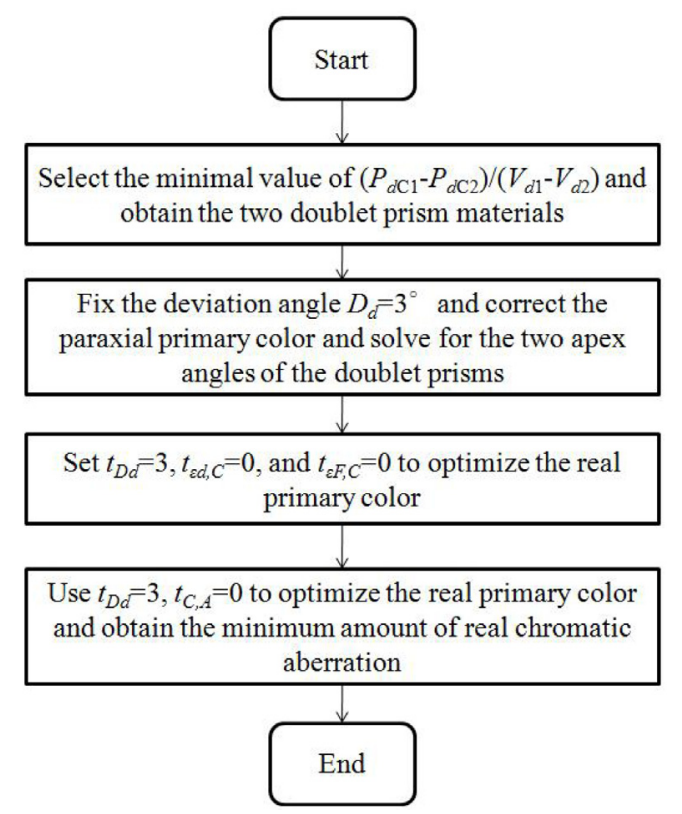

FIG. 7. Flow chart of optimization program for doublet prisms.

results for the optimized designs $\mathrm{A}$ to $\mathrm{F}$ are listed in Table 5. The chromatic aberration curves A to E corresponding to the optimal designs are shown in Fig. 8. This indicates that the proposed design method is effective in minimizing the chromatic aberration.

\section{CONCLUSION}

A low-cost optimal double-prism method combined with the developed MATLAB program to correct chromatic aberration has been presented. In comparison of the doublet-prism designs shown in Tables 1 and 5, shows that the areas between the paraxial and real chromatic aberration curves are similar. We can quickly find the best combination of doublet prisms by choosing the materials with small differences in relative partial dispersion and large differences in $\mathrm{V}_{\mathrm{d}}$ number, and minimizing the real

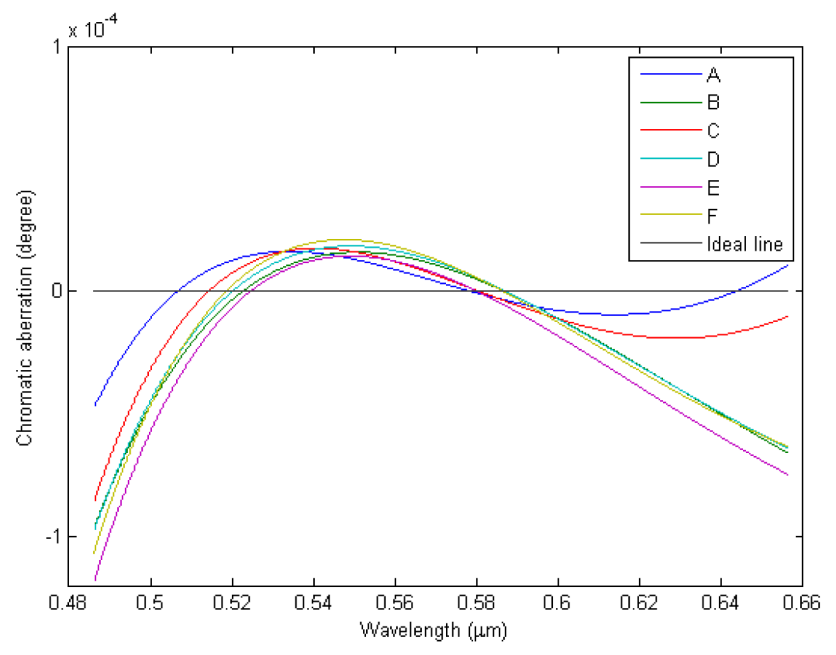

FIG. 8. Chromatic aberration curves A to E for the optimal designs.

chromatic aberration of doublet prisms by an optimization program.

\section{ACKNOWLEDGMENT}

The authors gratefully appreciate the support of the National Science Council of Taiwan, the Republic of China, under project numbers NSC 98-2221-E-008-021-MY3, NSC 100-2623-E-008-002-ET and NSC 101-2221-E-035-055.

\section{REFERENCES}

1. M. J. Kidger, Fundamental Optical Design (SPIE Press, Bellingham, WA, USA, 2001).

2. K. Seong and J. E. Greivenkamp, "Chromatic aberration measurement for transmission interferometric testing," Appl. Opt. 47, 6508-6511 (2008).

3. L. E. Sutton and O. N. Stavroudis, "Fitting refractive index data by least squares," J. Opt. Soc. Am. 51, 901-905 (1961).

4. R. E. Stephens, "Selection of glasses for three-color achromats," J. Opt. Soc. Am. 49, 398-401 (1959). 
5. P. N. Robb, "Selection of optical glasses. 1: two materials," Appl. Opt. 24, 1864-1877 (1985).

6. K. D. Sharma and S. V. Rama Gopal, "Design of achromatic doublets: evaluation of the double-graph technique," Appl. Opt. 22, 497-500 (1983).

7. J. L. Rayces, M. Rosete-Aguilar, "Selection of glasses for achromatic doublets with reduced secondary spectrum. I. tolerance conditions for secondary spectrum, spherochromatism, and fifth-order spherical aberration," Appl. Opt. 40, 5663- 5676 (2001).

8. J. L. Rayces and M. Rosete-Aguilar, "Selection of glasses for achromatic doublets with reduced secondary spectrum. II. application of the method for selecting pairs of glasses with reduced secondary spectrum," Appl. Opt. 40, 56775692 (2001).
9. S. Banerjee and L. Hazra, "Experiments with a genetic algorithm for structural design of cemented doublets with prespecified aberration targets," Appl. Opt. 40, 6265-6273 (2001).

10. E. Hecht, Optics, 4th ed. (Addision Wesley, New York, USA, 2002), p. 187.

11. D. Malacara and Z. Malacara, Handbook of Optical Design, 2nd ed. (Marcel Dekker, New York, USA, 2004), p. 253.

12. Schott, "Optical glass catalog," http://www.schott.com/advanced optics/ english/our_products/materials/.

13. D. P. Feder, "Automatic optical design," Appl. Opt. 2, 1209-1226 (1963).

14. C. G. Wynne and P. M. J. H. Worme, "Lens design by computer," Appl. Opt. 2, 1233-1238 (1963).

15. T. H. Jamieson, Optimization Techniques in Lens Design (American Elsevier, New York, USA, 1971). 